\title{
Mobility for an Optimal Data Collection in Wireless Sensor Networks
}

\author{
EZ-ZAIDI Asmaa \\ Laboratory of Applied Mathematics and \\ Computer Science (LAMAI) \\ Cadi Ayyad University \\ Marrakesh, Morocco
}

\author{
RAKRAK Said \\ Laboratory of Applied Mathematics and \\ Computer Science (LAMAI) \\ Cadi Ayyad University \\ Marrakesh, Morocco
}

\begin{abstract}
Sensor nodes located in the vicinity of a static sink drain rapidly their batteries since they have to carry more traffic burden. This situation results in network partition, holes as well as data losses. To mitigate this issue, many research proposed the use of mobile sink in data collection as a potential solution. However, due to its speed, the mobile sink has very short communication time to pick up all data from the sensor nodes within the network, therefore the sink is forced to return back to gather the remaining data. In this paper, we propose a new data collection scheme that aims to decrease the latency and enlarge the staying time between the mobile sink and the meeting points that buffer data originated from the other sensor nodes. We have also handled the case of urgent data so that they can be delivered without any delay. Our proposed scheme is validated via extensive simulations using NS2 simulator. Our approach significantly decreases the latency and prolongs the contact time between the mobile sink and sensor nodes.
\end{abstract}

Keywords-Contact time; mobile sink; wireless sensor networks; meeting point; data gathering

\section{INTRODUCTION}

The growth of the micro-electro-mechanical systems (MEMS) technology as well as wireless communications have resulted to the development of low-cost, low-power, multifunctional sensor nodes which are characterized by their small size and that communicate untethered over short distances. A wireless sensor network (WSN) is composed of hundreds of sensor nodes that are distributed over a large area to monitor and track physical phenomenon like humidity, temperature, sound and so forth. WSNs have a wide range of applications such as traffic monitoring [1], patient healthcare monitoring [2], target tracking [3], indoor living monitoring [4], localization [5], and many other interesting applications.

In traditional wireless sensor networks, collected data is forwarded by sensor nodes to a static base station via multihop routing. Nodes located near the static sink bear more traffic burden, consequently they drain their energy faster than the other nodes causing the hot spot problem [6], [7] (Fig. 1). The death of these nodes causes network disconnections, holes and data losses since the sink will be no more in connection with the rest of the network.

Sink mobility has been introduced in several works as a potential solution to overcome the issues cited above. The sink motion improves the network's performances such as energy, connectivity, reliability, security in data collection and many others benefits.

The remainder of this paper will be organized as follows: Section 2 presents some advantages and challenges of using sink mobility, Section 3 presents some related work, Section 4 describes the proposed scheme, Section 5 depicts the results of our scheme, conclusion and future works are presented in Section 6.

\section{ADVANTAGES AND CHALLENGES OF SINK MOBILITY IN DATA GATHERING}

\section{A. Enhancement of Network Lifetime}

Typical wireless sensor networks use multi-hop routing to transfer data toward the base station. Nonetheless, sensor nodes located in the sink's vicinity deplete their energy rapidly than the other nodes, leading to network degradation, disconnection as well as holes. Mobility was introduced to balance the energy consumption and minimize failures. The mobile sink moves in the network and pulls data buffered in sensor nodes that are within its communication range. By doing so, the multi-hop communication is reduced, and the traffic forwarding load is spread in the whole network. Several works have been conducted to decrease the network energy consumption by using sink mobility. For example, Shrivastava et al. [8] proposed a technique that consists on repositioning multiple mobile sinks towards regions with heavy traffic. Another work was introduced in [9], in which the authors examined how the mobility of sensor nodes operates in the wireless sensor network. They concluded that using mobile sensors results in better energy saving.

\section{B. Reliability}

As the number of hops increases, the probability of transmission errors as well as data losses increases too. A reliable data transmission is ensured by the use of mobile sink, this is because sensor nodes located near the sink's trajectory upload their data directly through fewer hops.

\section{Security}

Since the mobile sink keeps on changing its location around the sensor field, the chances of being attacked by external adversaries, and overhearing the collected information is decreased. In an attempt to avoid the injection of fake data, authors [10] proposed a random data collection scheme to protect the mobile sink from being tracked and 
becoming the target of attacks. The random motion of the sink keeps its location private and hard to track or predict. The mobile sink moves randomly around the network, and collects the sensed data stored in sensor nodes.

\section{Coverage and Connectivity}

Coverage has a great impact on WSN performances. It is one of the most important measurements in quality of service (Qos). Random deployment of sensor nodes, environmental disasters, presence of obstacles and power depletion of nodes all result in coverage holes. These holes may cause network dysfunction and interruption, which disturb the data collection process. Mobile sinks are used to overcome this problem; they visit disconnected regions where sensor nodes cannot operate to gather data. A tracking mechanism and a repairing robot algorithm were introduced in [11] to solve the coverage issue in the network. The moving robot carries a set of sensor nodes and performs hole-healing and repairs failure regions whenever it receives a request. In the same time, the mobile robot performs patrolling tasks to collect data.

Despite the various advantages that a mobile sink has nevertheless, many challenges may arise.

\section{E. Data Dissemination}

The communication overhead increases since the mobile sink has to broadcast each time its location within the network. Overhead causes energy wastage, that is why, it is important while designing data collection schemes to deal with this issue since sensor nodes have constrained resources.

\section{F. Obstacles}

The presence of obstacles inhibits the sink's movement in the network and degrades the communication quality between the sink and the sensor nodes.

\section{G. Data Delivery Latency and Packet Losses}

The data collection process depends on the contact time between the mobile sink and sensor nodes. When the sink moves at a high speed, it has very short time to pull data. Thus, sensor nodes have to wait for the sink to return, which prolong the delay of delivery. Moreover, an important packet losses and communication errors may occur due to the instability of the signal strength. Furthermore, since sensor nodes have limited buffer space, they may experience data losses, because they have to wait long time before the mobile sink comes in their vicinity again.

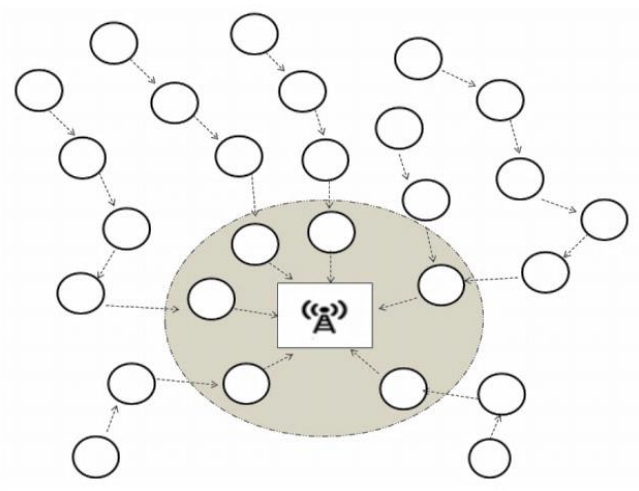

Fig. 1. The hotspot problem.

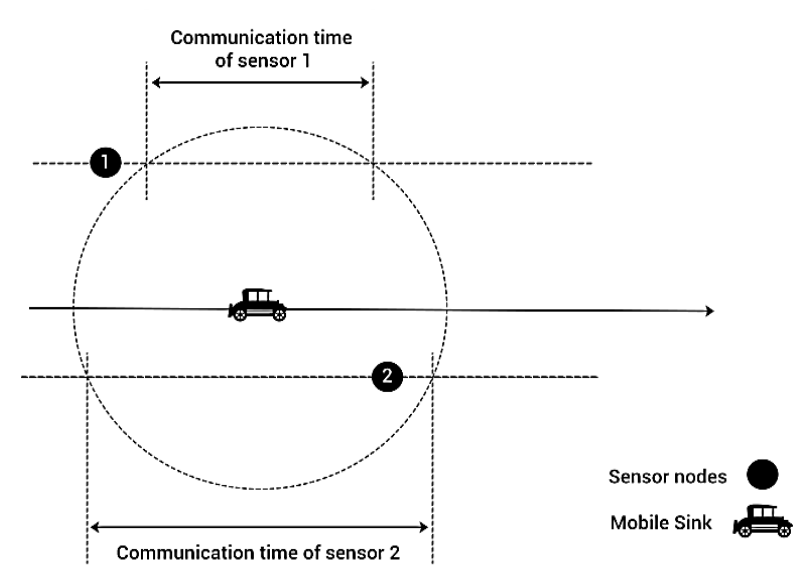

Fig. 2. Communication time between nodes and the sink.

\section{H. Communication Time}

Due to the high speed of the sink, the data collection process may be affected because of the short communication time between sensor nodes and the sink. As a consequence, only a small amount of data packets will be delivered to the mobile sink. So, nodes have to wait the return of the sink to complete the delivery of the rest of data. Fig. 2 shows the contact time between the mobile sink and the sensor nodes. Node 1 and 2 as instance can communicate with the sink from instant T1 to T2. However, node 2, which is located near the trajectory of the mobile sink, has relatively long contact time in comparison with node 1.

Aiming to decrease the latency while collecting a large amount of data, a data collection scheme is proposed in which a mobile sink travels the sensor network to pull data from Meeting points when it gets closer to them. These meeting points are special nodes that are elected according to some criteria, and have the ability to move when the mobile sink is within their range. Since we try to improve latency, we also took into consideration the case of urgent data that should be delivered in a fast manner.

Through this article, several questions will be answered:

- How to deliver maximum amount of data knowing beforehand that the communication time between the sensor nodes and the mobile sink is very limited? And in the same time avoid delays while collecting data.

- How to deliver urgent data effectively and without latency?

\section{RELATED WORK}

This section exposes some related works having used the mobility of the sink for an efficient data collection.

The use of mobile sinks (MSs) has been investigated in much research. Authors in [12], [13] have classified the MS motion according to three different types of mobility patterns, which are: Predictable, Random and Controlled.

In random mobility pattern, the base station is attached to entities that move in a random manner such as animals or people moving in a certain area. In this type of pattern, the 
mobile sink makes random movements in terms of direction and velocity. The controlled mobility refers to mobile devices that are guided in the network to accomplish specific tasks such as healing coverage, decreasing the energy consumption or repairing network failures. In the last type of mobility i.e. predictable that is used in our approach, the sink's movement can be predicted. In general, in this mobility pattern, the sink can be mounted on a public transportation such as busses, trains or vehicles, and follow a predetermined fixed path. By using this type of mobility, sensor nodes can anticipate the time of the sink's visit, and thus they can switch to sleep mode to save energy until the sink comes in their range.

In [14] authors studied the tradeoff between the energy conservation and data collection latency in wireless sensor networks. The approach's main concept consists in finding a set of special sensor nodes called polling points (PP) in such a way that all sensor nodes can send their data through a certain number of relay hops. The approach has demonstrated its effectiveness in shortening the tour length of the mobile collector.

$\mathrm{Xu}$ et al. [15] used a mobile sink travelling along a fixed trajectory to gather data without stopping. The sink, moving at a constant speed, collects data from gateway nodes that are located nearby its trajectory. These gateway nodes are relay nodes for the other sensors. Since the communication time with the gateway nodes is limited, the mobile sink cannot upload all the data sensed by nodes. To overcome this issue, data originated from only a subset of sensor nodes referred as packet nodes are collected by the sink and used to estimate those of the others. The maximum packet nodes are allocated to gateways having a large intersection time with the mobile sink.

A proactive data reporting protocol called SinkTrail was proposed by Liu et al. [16] in which the mobile sink moves in the field with a low speed, sojourns at some positions for a short time to collect data, and then moves to another location. Each position visited by the sink is viewed as a footprint. These footprints represent the logical coordinate of the sink in the network; they are used to guide the sensor nodes to report their data without knowing the physical locations as well as speed of the mobile sink. However, in a large scale network, huge delay may be induced especially for sensor located far away from the mobile sink.

A mobile sink based routing protocol "MSRP" was introduced in [17] in which the mobile sink visits locations of ClusterHeads having high residual energy to gather data. However, the approach may experience significant delays since the mobile sink favors zones of network that are rich in terms of resources, and overlook the other regions.

Authors in [18] proposed a Detour-Aware Mobile Sink Tracking (DAMST) for collecting data in a low overhead and in an energy efficient way. The mobile sink, while crossing a region, nominates a specific sensor node as a region agent called RA, which is in charge of gathering data around. In the same time, the sink records the location of nodes near its trajectory as footprints. When the sink needs information from the region agent, it constructs an energy efficient path from itself to that region; the path is established by analyzing the sink's movement angles and eliminating the footprints on detours by comparing adjacent movement angles.

Different from the approaches cited above, authors in [19] used a flying mobile sink that hover above a set of terrestrial sensors to collect data. The mobile sink is carried by a quadcopter because it is more flexible to move, descent and lift. Authors studied the speed, time of sojourning, flying trajectory, height of the sink as well as the amount of data to be transmitted to it.

Wang et al. [20] used several mobile sinks that move along the periphery of the network with a constant speed and sojourn at some particular parking positions for a certain time to gather data sensed from the sensor nodes. The next sojourn locations P1... Pn are picked from a PP_Table that stores all the parking positions. When the mobile sink is on its way to a parking position, it broadcasts a notification message to inform about its coming. Upon the movement of the mobile sink from a parking position $\mathrm{Pi}$ to $\mathrm{Pj}$ it will not collect data.

Authors in [21] used three mobile sinks to mitigate the problem of hot spots during data collection. The network is divided into two parts, an inner concentric circular region of radius $\mathrm{r}$ called area $\mathrm{A}$ and an outer circular region called region $\mathrm{B}$ that is divided into 8 portions $\mathrm{B} 1, \mathrm{~B} 2 \ldots \mathrm{B} 8$. One of the mobile sinks will span along the diameter of the circle, while the two others will travel along the arc line. These mobile sinks will move back and forth along their determined trajectories and sojourn at some fixed points to pick data.

In an attempt to collect the maximum amount of data, authors in [22] proposed a biased sink mobility scheme with adaptive pauses time for efficient data collection. When moving, the sink visits each region to gather data and adaptively stops for a time interval, which is proportional to the local data traffic. The introduction of pauses time increases the delivery success rate; however in large scale networks this scheme will incur important latencies while delivering data because of the large pauses made by the mobile sink. Furthermore, sensor nodes located in far regions will have to wait for a long time before the mobile sink comes again into their vicinity. This situation may cause buffer overflows since these nodes may not be able to hold all the generated data during this lapse of time.

Clearly by decreasing the mobile sink's velocity or by introducing the concept of stop times or favoring regions with high traffic density, the total amount of data collected will be improved, however the problem of latency will occur especially in large scale networks. In our approach, we do not need to lower the speed of the mobile sink or introduce pauses to collect large amount of data, or even visit regions having high density. We exploit the mobility of the sink as well as the meeting points so as to provide an efficient data collection without latency. We also take into account the priority of data when a set of nodes detects some alarming events. Our algorithm aims at optimizing the total amount of data collected and in the same time lowering the latency. 


\section{PROPOSED SOLUTION}

\section{System model}

Our sensor network as shown in Fig. 3 is composed of a set of sensor nodes deployed in a grid and a mobile sink that moves along a straight line.

- A set of sensor nodes denoted as SN deployed within the region of interest. These nodes generate data and relay it towards special nodes called meeting points according to multi-hop communication.

- Nodes located near the trajectory of the mobile sink (black dots) are called meeting points (MPs). In addition to sensing tasks, they are also responsible of delivering data at the mobile sink when this latter comes within range. MP nodes are used to enable the sink to collect a large amount of data without having to visit the whole network; this will minimize the delay caused by the long-distance that the sink travels.

- A mobile sink referred as MS, moves back and forth along a straight line at a constant speed. Its motion follows a predictable mobility model [23], which is used in various applications such as public transportation: buses and trains. We also assume that there are no obstacles that hinder the sink's movement.

The mobile sink is aware of its speed and trajectory, and all the sensor nodes within the network have the same communication range, and aware of their positions. Each sensor node generates the same amount of data.

The mobile sink has unlimited energy, memory and strong computational capacity. So, all the computations will be performed by the mobile sink.

- Proposed approach

The proposed data collection process is divided into several phases described below:

- First trip of the mobile sink

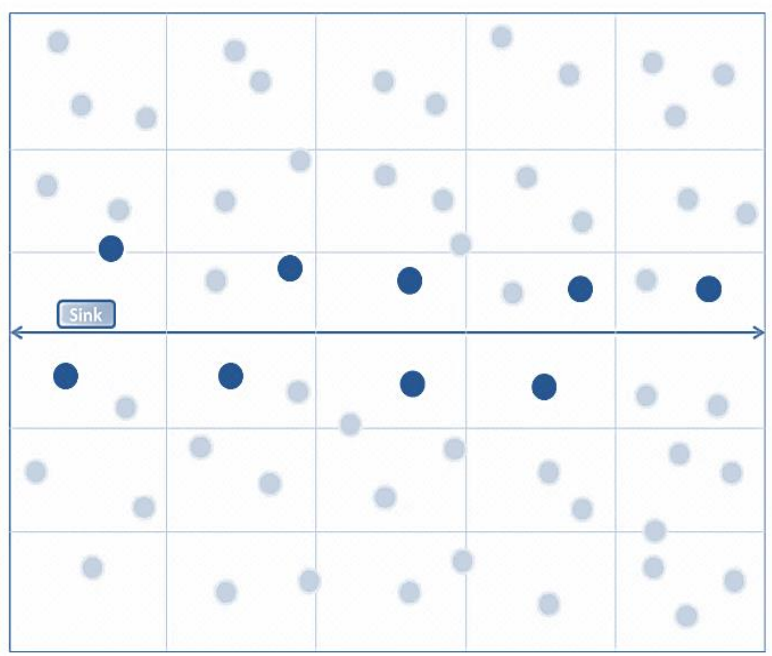

Fig. 3. Diagram of the wireless sensor network.
In its first trip, the mobile sink moves along the network and broadcasts periodically beacon messages to inform about its presence. Sensor nodes having received the broadcast send a message to the sink to express their candidacy to be meeting points. During this round, the mobile sink also records its communication duration with each sensor node.

- Second trip (Election of meeting points)

In this trip, the mobile sink travels the trajectory for a second time to broadcast results about the elected meeting points. The computation of the most suitable nodes to be nominated as MPs is done by the mobile sink since it has very strong power computations. In fact, the meeting points guarantee the non-disconnection of the network since they act like a bridge between the mobile sink and the other sensors within the network. They buffer incoming data until the sink passes by to collect it.

The mobile sink elects the most convenient nodes to be meeting points; they should have a high energy level, and located near the sink's trajectory to deliver data reliably and within one hop, and finally they must have a large intersection time with the mobile sink so as to transfer important amount of data as shown in Fig. 4.

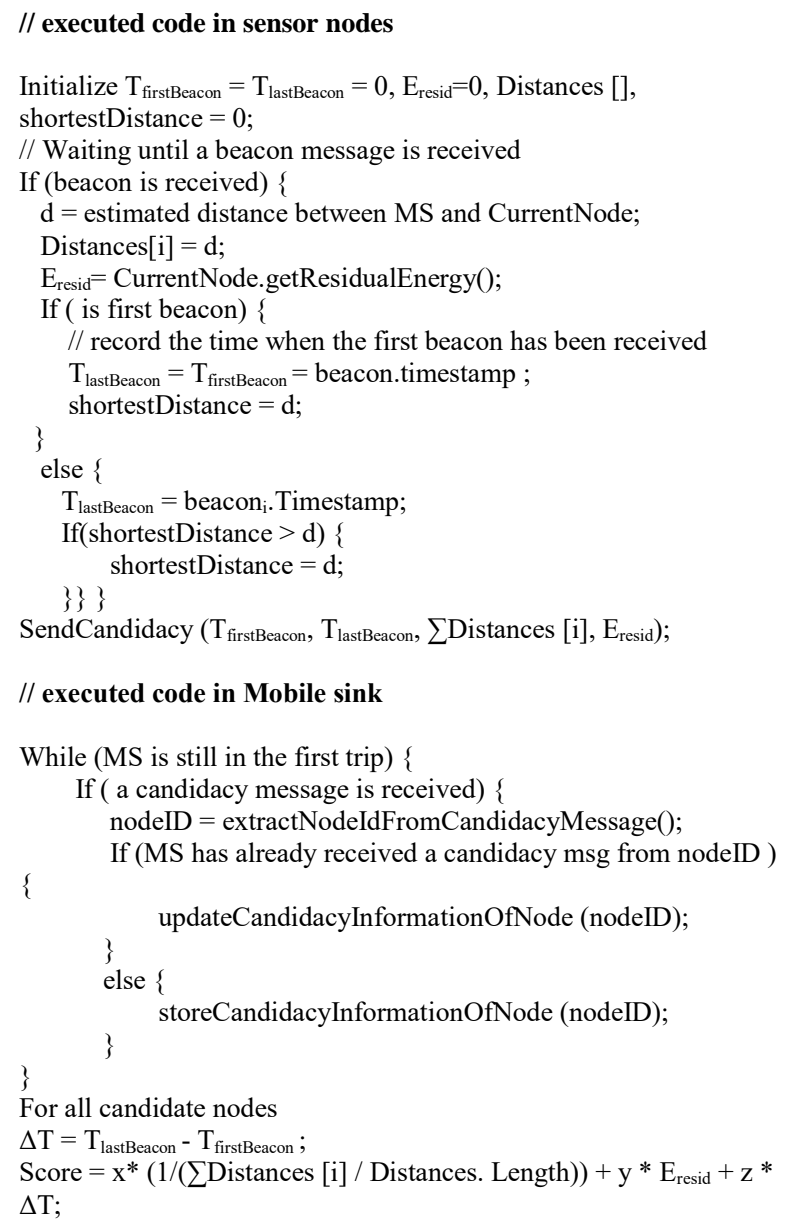

Fig. 4. Depicts the algorithm of meeting points' election. 
The parameters of choosing the meeting points are based on [24]. After the reception of candidacies, the mobile sink calculates the cost value of each candidate node according to the algorithm below and sorts it in an increasing order. Elected nodes are those who have the highest cost value. Finally, the sink informs each chosen node through a message about its election. Once elected, each meeting point builds the shortest path rooted from them. That way, each sensor node can relay its collected data towards the meeting point to which it is attached.

$\mathrm{X}, \mathrm{Y}$ and $\mathrm{Z}$ are coefficients of the distance, energy level and time of communication, respectively.

\section{- Data collection}

After the meeting points' election, the data collection process begins. The meeting points are used because they enable the mobile sink to pull data without having to visit the entire network. They serve as data collection points for many sensor nodes. Furthermore they play a major role in decreasing the data collection delay.

Each sensor node forwards its sensed data, using multi-hop communication to its attached meeting point, this latter stores data until the arrival of the mobile sink to fetch it. Since the mobile sink has a very short time to communicate with the meeting points due to its speed, so only few amounts of data will be collected. Besides, when the size of data is important that it cannot be entirely delivered during the contact time, the MPs have to wait for the sink to return again which induces large latencies. In an attempt to find a compromise between collecting a large volume of data, and in the same time avoiding long delays in data gathering, our proposal consists on moving the meeting points for a definite distance with the mobile sink to remain the longest possible in contact so as to deliver maximum quantity of data.

Upon its movement back and forth along the path, the mobile sink sends periodically a Notification_Message that contains information about its speed, position as well as its direction (forth or back) to announce its presence. As the mobile sink enters the communication range of the meeting point, the data collection process begins (Fig. 5(a)).
When the signal strength (shortest Distance) between the mobile sink and the meeting point reaches its peak, the meeting point starts moving for a certain distance parallel to the sink (Fig. 5(b)) with the aim to prolong the staying time between each other.

The distance travelled by the meeting point is equal to $2 / 3$ * $\mathrm{d}$ where $\mathrm{d}$ is the distance between two consecutive sensor nodes within the grid. In fact, the choice of such distance is to avoid collisions and the overlap that may occur between the meeting points that try to deliver data simultaneously to the mobile sink.

After crossing the specified distance, the meeting point stops and wait for the sink to come back. When the latter returns, the MP will move again another $2 / 3 * d$ to return back to its initial position (start position). By doing so, we ensure that more packets are delivered to the mobile sink without having to make stops.

\section{- Priority of data}

We considered two types of data, the normal ones (temperature) and high-priority ones (detection of a critical event) that must be sent within a shorter delay. All along the data collection, each meeting point filters the received data packets according to a flag to distinguish between sensitive and normal ones.

When the mobile sink enters within range, the urgent data are first delivered then the normal ones. In case, the size of the priority data is big enough and cannot be gathered completely by the sink within the contact time, the meeting point forwards the rest of it toward the future location of the mobile sink to avoid any delays.

To do so, the current MP (the meeting point that possesses the priority data) looks for a near meeting point according to the sink's direction movement (forward or backward). If the meeting point is located within its communication range, the rest of priority data is sent directly to it, otherwise multi-hop communication via relay nodes is used to relay the remaining data as depicted in Fig. 6. By doing so, it is guaranteed that data that has priority reaches the sink in a timely manner. Fig. 7 depicts the algorithm of the data collection process.

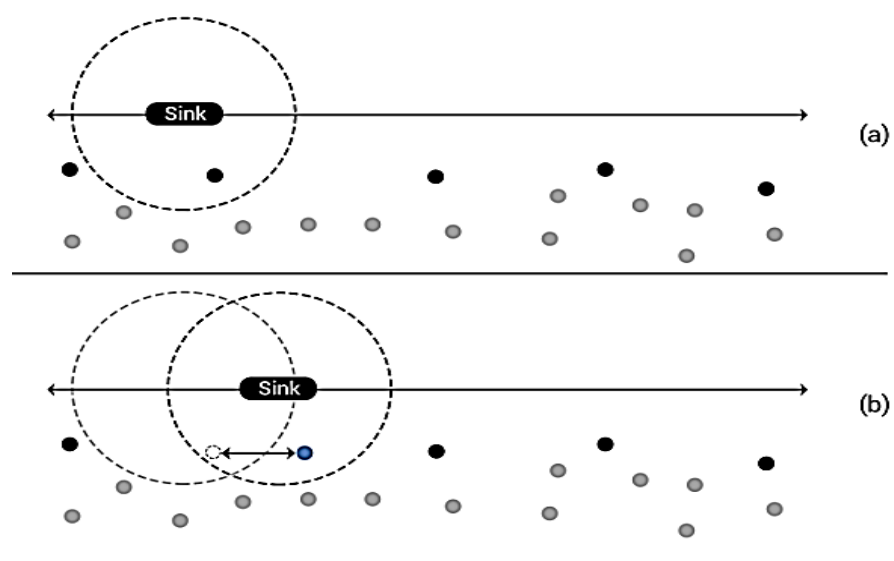

(a)

(b)

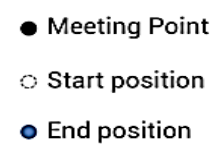

Fig. 5. Meeting point movement. 


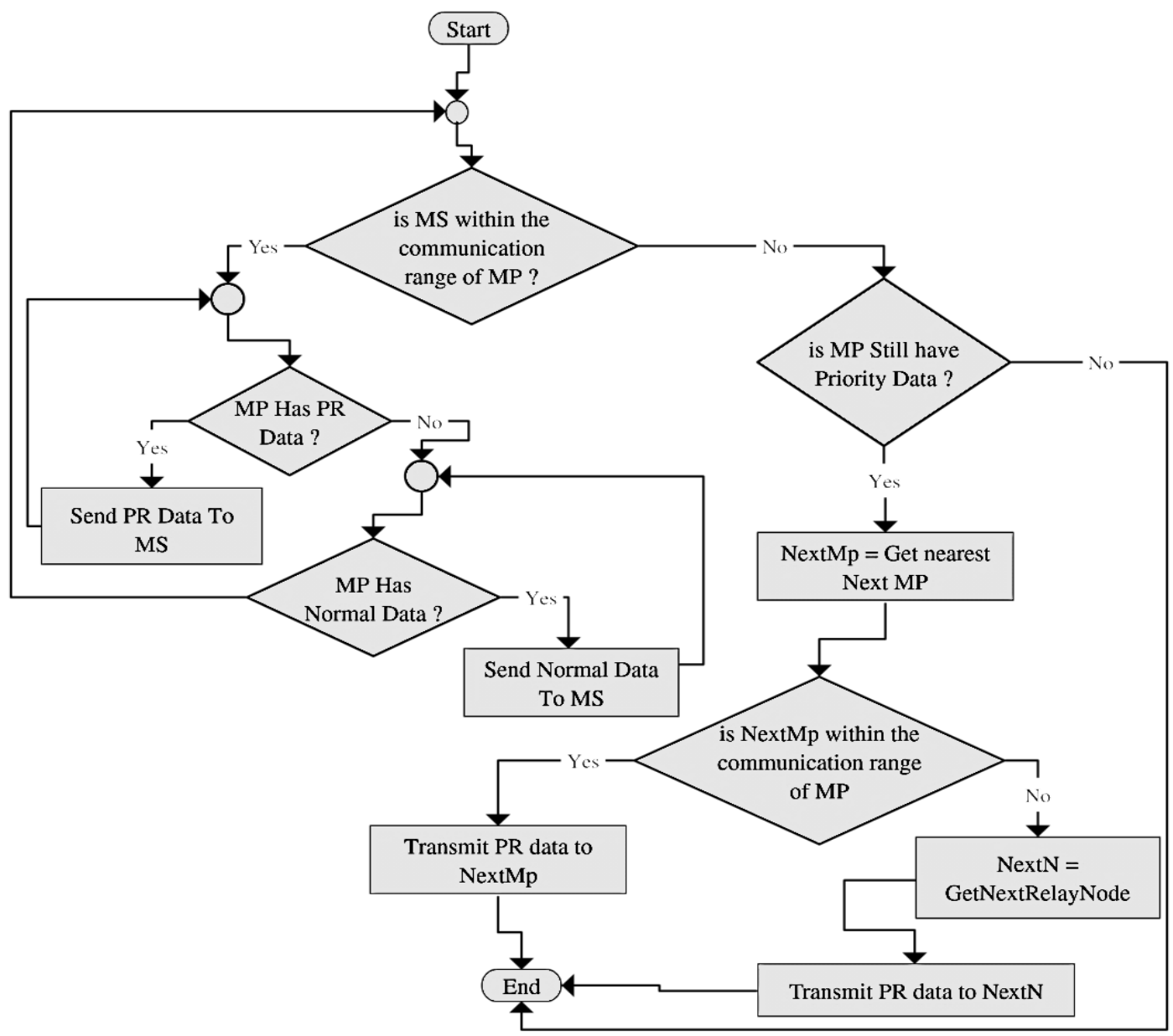

Fig. 6. Priority of data diagram.

Initialize V_MP, V_MS, Pr, isMoving = false;

// the variables are respectively: velocity of MP, MS, priority and a Boolean to check whether the MP is moving or not

While (First NotificationMsg has not been received yet) \{ MP waits in its actual position;

MP collects data from sensor nodes; \}

MP performs filter based on data priority flag;

// First Notification message is received

V_MP $=$ V_MS // set the velocity of the MPs

While (MS is still within range of MP) \{

D = estimatedDistanceBetweenMP_And_MS ();

If $(\mathrm{D}<=$ shortestDistance $-\mu)\{$ If (!isMoving) \{ Start moving $2 / 3 * \mathrm{~d}$;

\} \} isMoving = true;

If $(\mathrm{Pr}) \quad$ // priority data exists. Transfer Priority data ();

Else

Transfer non-priority data ();

\}

If (Still Pr data) \{ // MS is out of range (timeout expired) Send remain priority data to the Next MP (cf. figure 6) \}

isMoving $=$ false;

\section{PERFORMANCE EVALUATION}

To further evaluate the efficiency of the proposed approach, we have used NS-2.35 which is a discrete event simulator.

\section{A. Simulation setup}

We have considered a $200 * 200$ network area; the number of sensor nodes is 300. All the sensor nodes have the same communication range $\mathrm{R}=15 \mathrm{~m}$. Each sensor node generates at random times about 1 message per 10 seconds and sends it to its parent. The simulation time is 2500 seconds. The approach is evaluated by considering different speeds of the mobile sink which are 4, 10 and $20 \mathrm{~m} / \mathrm{s}$.

Number of mobile sinks: 1 mobile sink.

Number of nodes: 300.

Simulation area: $200 * 200 \mathrm{~m}$

Speed of the mobile sink: 4, 10 and $20 \mathrm{~m} / \mathrm{s}$.

Communication range: $15 \mathrm{~m}$

Simulation runtime: $2500 \mathrm{~s}$

Mac protocol 802.15.4

Fig. 7. Data collection algorithm. 
We have considered three parameters for evaluation which are data latency, packet delivery ratio and contact time duration.

- Packet delivery ratio represents the ratio of packets which are successfully delivered to the sink compared to the total packets that have been generated.

- Data latency: the time elapsed between the creation of the message and its delivery to the sink.

- Contact time duration: The communication time between the mobile sink and the meeting points.

The simulation was run several times; we took the average of the runs to show the results.

\section{B. Findings}

1) Duration of the communication: Fig. 8 shows how the mobility of the meeting points increases the contact time duration while gathering data.

As discussed above, the limited communication time between sensor nodes and the mobile sink impacts the data collection process since only small amount of data will be gathered.

We notice from the graph that when the meeting points are static the contact duration with the sink is relatively very short, therefore the MPs upload only few amount of data packets and have to wait until the sink returns back to upload the rest of data. However, when the meeting points are mobile the communication time is enlarged, so in one hand more data is collected and in the other hand the latency will be minimized as shown in the next graph (Fig. 9).

2) Latency: Fig. 9 shows the latency of the network for different speeds of the mobile sink. As expected, when moving the meeting points, our scheme achieves a much better performance in terms of latency in all cases of sink's velocity $\{4,10$ and $20 \mathrm{~m} / \mathrm{s}\}$.

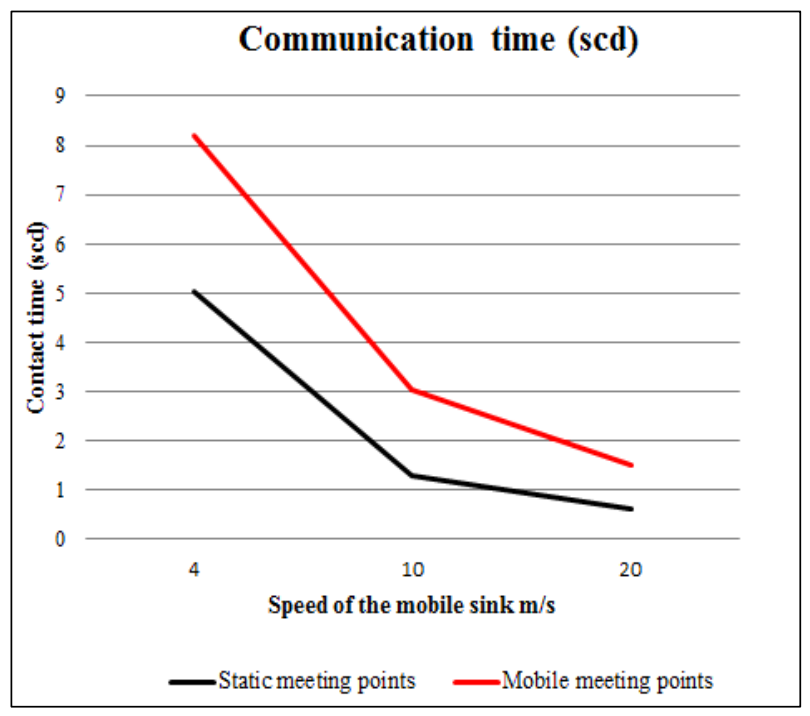

Fig. 8. Communication time.
Conversely, the latency is very high in the case of static meeting points. This goes back to the fact that the communication time between the mobile sink and the meeting points is very short, thereby data is not fully collected. So, the sink is forced to make several trips back and forth to gather the rest of packets. This situation obviously increases the latency.

It is also noticed that latency is slightly high when the sink' velocity is $4 \mathrm{~m} / \mathrm{s}$ or $20 \mathrm{~m} / \mathrm{s}$. With a speed of $4 \mathrm{~m} / \mathrm{s}$, the MS moves slowly and takes a long time to collect data within the network. With a speed of $20 \mathrm{~m} / \mathrm{s}$, the sink moves at a high speed which reduces the contact time. The sink is forced to return repeatedly to collect data buffered in the meeting points. Conversely, the mobile sink takes less time to visit all the meeting points when it moves with $10 \mathrm{~m} / \mathrm{s}$. The speed of $10 \mathrm{~m} / \mathrm{s}$ balances between latency and quantity of collected data.

3) Packet delivery ratio (\%): In Fig. 10 the number of collected data using static meeting points is almost equal to the case of mobile meeting points.

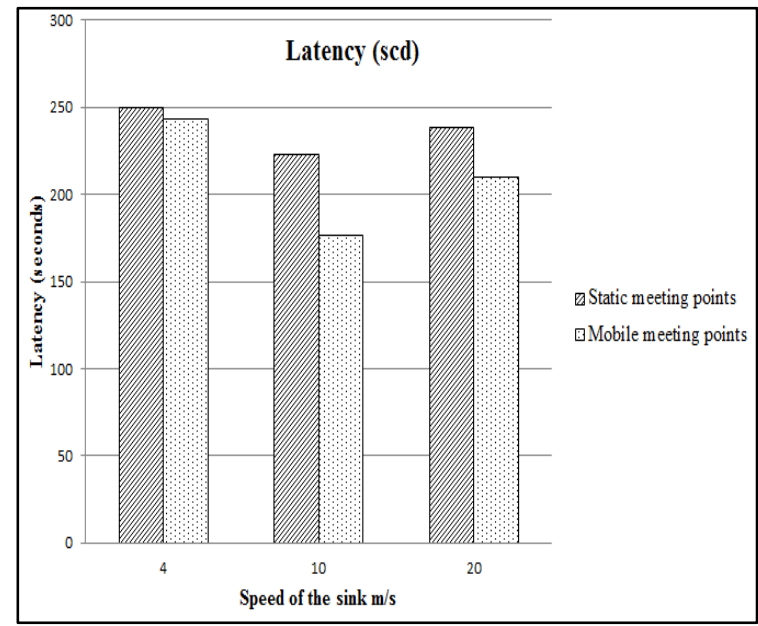

Fig. 9. Latency.

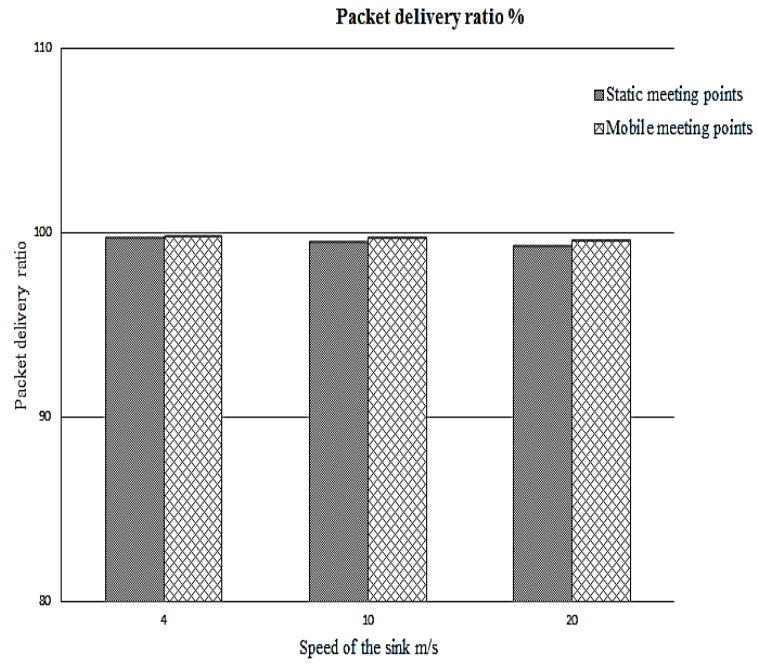

Fig. 10. Packet delivery ratio. 
We used the mobility of meeting points to ensure a maximum contact time with the sink which increase the quantity of collected data. The plus in our approach is that we have achieved a balance between how to have a high packet delivery ratio while reducing the latency. From the figure below, the number of collected packets is high regardless of the sink's speed. When the mobile sink spans the network at a speed of $20 \mathrm{~m} / \mathrm{s}$, the success rate is slightly low in comparison with the speed of $4 \mathrm{~m} / \mathrm{s}$ and $10 \mathrm{~m} / \mathrm{s}$. The high speed of the sink causes packet drops which subsequently impact the ratio of delivered packets.

\section{CONCLUSION AND PERSPECTIVES}

In this paper, an efficient data collection using the mobility of both the mobile sink and the meeting points was proposed. Our approach aims to collect large amount of data in a very short time without having to travel the whole network or make pauses time. Urgent data are also handled while collecting data, they are delivered without any delay.

Simulation results have shown that we achieve a high success rate without delays. Our future work will focus on the energy aspect; the frequent mobility of meeting points consumes much energy, as well as the retransmission of packets.

\section{REFERENCES}

[1] Bottero, M., Dalla Chiara, B., Deflorio, F. P. (2013). Wireless sensor networks for traffic monitoring in a logistic centre. Transportation Research Part C: Emerging Technologies, 26, 99-124.

[2] Manvi, S. S., Manjula, R. B. (2014). A Survey on Health Care Services Using Wireless Sensor Networks. Healthcare Administration: Concepts, Methodologies, 132.

[3] Ez-Zaidi, A., \& Rakrak, S. (2015). A Comparative Study of Target Tracking Approaches in Wireless Sensor Networks. Journal of Sensors, 2016.

[4] M. Bhuiyan and G. Wang, "Deploying wireless sensor networks with fault-tolerance for structural health monitoring," Computers, IEEE Trans., 2015.

[5] Halder, S., \& Ghosal, A. (2015). A survey on mobile anchor assisted localization techniques in wireless sensor networks. Wireless Networks, $1-20$.

[6] Kuo, C. H., Chen, T. S., \& Lo, Y. H. (2015). Efficient traffic load reduction algorithms for mitigating query hotspots for wireless sensor networks. International Journal of Ad Hoc and Ubiquitous Computing, 18(3), 153-163.

[7] Jannu, S., \& Jana, P. K. (2016). Energy Efficient Algorithms for Hot Spot Problem in Wireless Sensor Networks. In Proceedings of the Second International Conference on Computer and Communication Technologies (pp. 509-517). Springer India.

[8] Shrivastava, P., Pokle, S.B.: A multiple sink repositioning technique to improve the energy efficiency of wireless sensor networks. 2013 15th
International Conference on Advanced Computing Technologies (ICACT). pp. 1-4. IEEE (2013).

[9] Yang, T., Mino, G., Barolli, L., Durresi, A., \& Xhafa, F. (2011, June), Energy-saving in wireless sensor networks considering mobile sensor nodes. In Complex, Intelligent and Software Intensive Systems (CISIS), 2011 International Conference on (pp. 249-256). IEEE.

[10] Ngai, E., Rodhe, I.: On providing location privacy for mobile sinks in wireless sensor networks. Wirel. networks. (2013).

[11] Chang, C.-Y., Lin, C.-Y., Yu, G.-J., Kuo, C.-H.: An energy-efficient hole-healing mechanism for wireless sensor networks with obstacles. Wirel. Commun. Mob. Comput. 13, 377-392 (2013).

[12] Tunca, C., Isik, S.: Distributed mobile sink routing for wireless sensor networks: a survey. Communications Surveys \& Tutorials, IEEE. (2014).

[13] Khan, M., Gansterer, W., Haring, G.: Static vs. mobile sink: The influence of basic parameters on energy efficiency in wireless sensor networks. Comput. Commun. (2013).

[14] Zhao, M., and Yang, Y. (2012). Bounded relay hop mobile data gathering in wireless sensor networks. Computers, IEEE Transactions on, 61(2), 265-277.

[15] Xu, X., Liang, W., and Wark, T. (2011, June). Data quality maximization in sensor networks with a mobile sink. In Distributed Computing in Sensor Systems and Workshops (DCOSS), 2011 International Conference on (pp. 1-8). IEEE.

[16] Liu, X., Zhao, H., Yang, X., and Li, X. (2013). SinkTrail: a proactive data reporting protocol for wireless sensor networks. Computers, IEEE Transactions on, 62(1), 151-162.

[17] Nazir, B., and Hasbullah, H. (2010, December). Mobile sink based routing protocol (MSRP) for prolonging network lifetime in clustered wireless sensor network. In Computer Applications and Industrial Electronics (ICCAIE), 2010 International Conference on (pp. 624-629). IEEE.

[18] Yang, G., Xu, H., He, X., Wang, G., Xiong, N., \& Wu, C. (2016). Tracking Mobile Sinks via Analysis of Movement Angle Changes in WSNs. Sensors, 16(4), 449.

[19] Chen, Y., Chen, J., Zhou, L., Du, Y.: A data gathering approach for wireless sensor network with quadrotor-based mobile sink node. Adv. Wirel. Sens.Networks. (2012).

[20] Wang, J., Li, B., Xia, F., Kim, C. S., \& Kim, J. U. (2014). An energy efficient distance-aware routing algorithm with multiple mobile sinks for wireless sensor networks. Sensors, 14(8), 15163-15181.

[21] Wang, J., Zuo, L., Shen, J., Li, B., and Lee, S. (2015). Multiple mobile sink based routing algorithm for data dissemination in wireless sensor networks. Concurrency and Computation: Practice and Experience, 27(10), 2656-2667.

[22] Kinalis, A., Nikoletseas, S., Patroumpa, D., and Rolim, J. (2014). Biased sink mobility with adaptive stop times for low latency data collection in sensor networks. Information fusion, 15, 56-63.

[23] Dong, Q., Dargie, W.: A Survey on Mobility and Mobility-Aware MAC Protocols in Wireless Sensor Networks. IEEE Commun. Surv. Tutorials. $15,88-100$ (2013).

[24] Konstantopoulos, C., Pantziou, G., Gavalas, D., Mpitziopoulos, A., Mamalis, B.: A Rendezvous-Based Approach Enabling Energy-Efficient Sensory Data Collection with Mobile Sinks. IEEE Trans. Parallel Distrib. Syst. 23, 809-817 (2012). 